Chirurg 2013 $\cdot 84: 906$

DOI 10.1007/s00104-013-2615-8

Online publiziert: 27. September 2013

๑) Springer-Verlag Berlin Heidelberg 2013
L. Haeder · J. Jähne

Klinik für Allgemein- und Viszeralchirurgie, Schwerpunkt für endokrine und onkologische Chirurgie, Diakoniekrankenhaus Henriettenstiftung gGmbH, Hannover

\title{
Einfluss der gastrojejunalen Rekonstruktion nach Pankreatikoduodenektomie auf die Magenentleerung
}

\section{Orginalpublikation}

Shimoda M, Kubota K, Katoh M, Kita J (2013) Effect of billroth II or Roux-en-Y reconstruction for the gastrojejunostomy on delayed gastric emptying after pancreaticoduodenectomy. A randomized controlled study. Ann Surg 257:938-942

\section{Hintergrund}

Mit der Verbesserung operativer Techniken und des perioperativen Managements in der Behandlung pankreatischer oder periampullärer Tumoren konnte eine $\mathrm{Re}$ duktion der postoperativen Mortalitätsrate auf unter $5 \%$ erreicht werden. Allerdings liegt die Morbidität immer noch bei 30-60\%, wobei die Magenentleerungsstörung (DGE) als eine wesentliche Majorkomplikation angesehen wird, die entscheidenden Einfluss auf den postoperativen Kostaufbau, die stationäre Verweildauer und die damit korrelierenden Behandlungskosten hat. Obwohl frühere Untersuchungen intraabdominelle Komplikationen als ursächlich für die DGE ansehen, wird die für das Outcome optimale Resektions- sowie Rekonstruktionstechnik immer noch kontrovers diskutiert. Daher war es das Ziel dieser randomisierten kontrollierten Studie, den Einfluss der Rekonstruktionstechnik (Billroth II [B II] vs. Roux-Y) der Gastrojejunostomie (GJ) auf die Inzidenz des postoperativen DGE nach Pankreatikoduodenektomie zu evaluieren.

\section{Methoden und Ergebnisse}

Zwischen 2008 und 2011 wurden insgesamt 101 Patienten mit einem periampul- lären oder Pankreaskopftumor mittels subtotaler magenerhaltender Pankreatikoduodenektomie (SSPPD) nach Randomisierung in die B-II-Gruppe $(\mathrm{n}=52)$ und die Roux-Y-Gruppe $(n=49)$ operiert. Die Rekonstruktion erfolgte jeweils als antekolische End-Seit-Gastro-Jejunostomie mit einer Braun-Fußpunktanastomose oder als End-Seit-Gastro-Jejunostomie nach Roux-Y. Primärer Endpunkt der Studie war die DGE-Inzidenz, wobei hier die Klassifikation der International Study Group of Pancreatic Surgery (Grade A-C) angewendet wurde.

Die Gesamtinzidenz der Magenentleerungsstörung zeigte sich in der B-II-Gruppe signifikant geringer als in der Roux-YGruppe (5,7\% vs. 20,4\%). Hinsichtlich des Schweregrades war der Unterschied bei DGE Grad C deutlicher (1,9\% vs. 16,3\%) als bei DGE Grad B (3,8\% vs. $4 \%$ ). Damit einhergehend war die Rate an notwendiger Neuplatzierung der nasogastralen Sonde geringer (5,7\% vs. $18,7 \%)$ und die Phase rein flüssiger Kostzufuhr kürzer $(8,5 \pm 3,3$ Tage vs. $12,0 \pm 8,0$ Tage $)$. Auch die stationäre Verweildauer war in der B-IIGruppe signifikant kürzer $(31,6 \pm 15,0$ Tage vs. $41,4 \pm 20,5$ Tage, $p=0,037)$. Die Rate an Pankreasfisteln unterschied sich nicht signifikant in beiden Gruppen, allerdings konnte in einer univariaten Analyse die DGE als Risikofaktor für die Entwicklung einer Pankreasfistel detektiert werden.

\section{Diskussion und Fazit}

Obwohl die genauen pathophysiologischen Mechanismen der postoperativen Magenentleerungsstörung nicht abschließend geklärt sind, haben sich be- reits verschiedene Untersuchungen mit der Frage beschäftigt, inwiefern unterschiedliche Resektions- und Rekonstruktionstechniken die DGE-Rate senken können, wobei die antekolische Duodenojejunostomie bzw. Gastrojejunostomie vorteilhaft zu sein scheint. Die japanischen Autoren argumentieren, dass sich aus früheren prospektiven Studien, welche die DGE-Rate in Bezug auf die chirurgische Technik untersucht haben, keine eindeutigen Empfehlungen ableiten lassen, sodass in dieser erstmals randomisiert kontrollierten Studie eine Überlegenheit der B-II-Rekonstruktion verglichen mit der Roux-Y-Rekonstruktion nachzuweisen war, wobei sich diese allerdings nur auf die SSPPD bezog. Daher müssten weitere Untersuchungen auch die übrigen Rekonstruktionsvarianten inklusive prozeduraler Komplikationen mit erfassen, um hier vielleicht eine eindeutige Aussage zur optimalen Rekonstruktionstechnik tätigen zu können, sodass sich eine Reduktion der postoperativen Morbidität mit ihrer hohen sozioökonomischen Relevanz erreichen ließe.

\section{Korrespondenzadresse}

\section{Haeder}

Klinik für Allgemein- und Viszeralchirurgie, Schwerpunkt für endokrine und onkologische Chirurgie, Diakoniekrankenhaus Henriettenstiftung $\mathrm{gGmbH}$, Marienstr. 72-90, 30171 Hannover Lars.Haeder@ddh-gruppe.de

\section{Einhaltung ethischer Richtlinien}

Interessenkonflikt. L. Haeder und J. Jähne geben an, dass kein Interessenkonflikt besteht. 